\title{
DISPOSICIÓN A PAGAR POR EL SERVICIO DE UN TREN RÁPIDO ENTRE BARRANQUILLA Y CARTAGENA
}

\section{- LuIS GABRIEL MÁRQUEZ DíAZ ${ }^{1}$}

\section{RESUMEN}

El presente trabajo estudia los factores que influyen en la disposición a pagar (DAP) por un nuevo servicio de tren rápido de pasajeros entre Barranquilla y Cartagena. Como instrumento de toma de datos se utilizó la técnica de valoración contingente y mediante una estrategia de experimentación secuencial fueron identificados los factores con efecto significativo sobre la DAP, cuyo valor medio fue de \$29.000. Se calibró un modelo de regresión de primer orden que explica la DAP en función de las variables intervalo del servicio, tiempo de viaje y acceso a red Wi-Fi y tomas eléctricas. El proceso de optimización para la variable de respuesta permitió aumentar su valor hasta \$39.000, que se considera muy bajo si se compara con los referentes internacionales para este tipo de servicios, llegando a la conclusión que queda en entredicho la viabilidad del sistema propuesto.

PALABRAS CLAVE: Disposición a pagar; tren de alta velocidad; análisis de varianza; regresión lineal múltiple.

\section{WILLINGNESS TO PAY FOR A HIGH-SPEED PASSENGER RAIL SERVICE BETWEEN BARRANQUILLA AND CARTAGENA}

\section{ABSTRACT}

This paper shows the factors influencing the willingness to pay (WTP) for a new high-speed passenger rail service between Barranquilla and Cartagena. We used the contingent valuation as an instrument for data collection and we conducted a sequential experimental design in order to identify factors that have significant effect on DAP, which average value was $\$ 29,000$ Colombian pesos. We calibrated a first order regression model to explain the DAP based on the variables: headway, travel time and Wi-Fi availability and electrical outlets. The optimization process greatly enhanced the DAP to a cost of $\$ 39,000$ Colombian pesos, which is considered very low compared with international benchmarks for these services, concluding that it calls into question the viability of the proposed system.

KEYWORDS: Willingness to pay; High-speed passenger rail service; ANOVA; Multiple Linear Regression.

1 Transportation and Highways Engineering. MSc. in Transportation Engineering. Associate professor at Universidad Pedagógica y Tecnológica de Colombia.

Autor de correspondencia: Márquez Díaz, L.G. (Luis Gabriel). Avenida Central del Norte 39-115 Sede Central Tunja-Boyacá-Colombia / Tel.: (57+8) 7405626 Correo electrónico: lgmarquezd@gmail.com
Historia del artículo:

Artículo recibido: 07-V-2016/ Aprobado: 20-XI-2016

Disponible online: 30 de febrero de 2017

Discusión abierta hasta abril de 2018 


\section{DISPOSIÇÃO A PAGAR POR UM SERVIÇO FERROVIÁRIO DE ALTA VELOCIDADE PARA PASSAGEIROS ENTRE BARRANQUILLA E CARTAGENA}

\section{RESUMO}

Este artigo mostra os fatores que influenciam na disposição a pagar (DAP) para um novo serviço ferroviário de alta velocidade para passageiros entre Barranquilla e Cartagena. Foi utilizada a avaliação contingente como instrumento de coleta de dados e mediante uma estratégia de experimentação sequencial, foram identificados os factores com efeito significativo sobre a DAP, cujo valor médio foi de \$29,000 pesos colombianos. Fio calibrado um modelo de regreção de primeira ordem que explica a DAP em função das variáveis de intervalo do serviço, tempo de viagem e acesso a Wi-Fi e tomadas elétricas. 0 processo de otimização para a variável de resposta permitiu aumentar seu valor até 39,000 pesos colombianos, o que é considerado muito baixo em comparação com os referentes internacionais para esses serviços, chegando à conclusão da viabilidade do sistema proposto.

PALAVRAS-CHAVE: Disposição a pagar; Serviço ferroviário de alta velocidade para passageiros; Análises de varianza; Regreção Linear Múltipla.

\section{PLANTEAMIENTO}

Un experimento puede entenderse como una serie de pruebas, donde se presentan cambios controlados a través de las variables de entrada de un proceso o sistema con el fin de identificar las razones de los cambios que se observan en la variable de salida (Montgomery, 2013). A pesar de que la mayoría de las aplicaciones de diseño y análisis de experimentos se encuentran en el sector industrial (Eriksson et al., 2008), es evidente que su alcance incluye analizar un sinnúmero de fenómenos tales como combustible de automoción, precio al por mayor de productos químicos, o el rendimiento del maíz por acre (Goupy y Creighton, 2007).

En el marco de la ingeniería de transporte se ha utilizado el diseño y análisis de experimentos en diferentes contextos. Por ejemplo, para analizar el impacto del uso de sistemas de transporte terrestre inteligentes en redes de distribución de mercancías según carretera (Polo-Navarro et al., 2014), para estudiar la fiabilidad y la seguridad de los sistemas de transporte marítimo (Xie et al., 2012) o para explorar los conceptos de capacidad, eficiencia y flexibilidad de los sistemas de transporte aéreo (Wilson, 2011).

En los últimos años, los legisladores en Europa y Estados Unidos han considerado el desarrollo de infraestructuras ferroviarias de alta velocidad como una posible estrategia para reducir los impactos climáticos del sector de transporte (Clewlow et al., 2014). El crecimiento del sistema ferroviario de alta velocidad ha dado lugar a importantes cambios en la oferta del transporte interurbano en aquellos países que han extendido sus redes y servicios (Albalate et al., 2015). La infraestructura ferroviaria también ha experimentado un crecimiento próspero en China debido a una gran inversión del gobierno que benefició a su sociedad y su economía, gracias a la mejora en la eficiencia del transporte ferroviario y la accesibilidad regional (Chen et al., 2016).

Es evidente que el transporte afecta al medio ambiente de muchas maneras. La reducción de los factores ambientales externos es un beneficio potencial de la inversión en un sistema ferroviario de alta velocidad, aunque esto depende del volumen de la demanda que difiere de las modalidades de transporte menos amigables con el medio ambiente, y si la demanda es lo suficientemente alta para compensar las 
externalidades negativas durante la construcción, el efecto barrera, el ruido y la intrusión visual (de Rus y Kågeson, 2012). En el caso colombiano, se ha demostrado la importancia de incluir los costos externos en la adaptación estratégica de las redes interregionales de transporte de mercancías como parte de una política que apoya los mecanismos para la gestión y la fijación de precios con el fin de alcanzar el óptimo social (Márquez y Cantillo, 2013).

La planificación para el servicio ferroviario de cercanías o interurbano de pasajeros abarca una amplia gama de actividades, desde el ajuste de configuraciones de servicio (por ejemplo, frecuencias y servicios a bordo) hasta el establecimiento de nuevas rutas en las que no existe servicio actualmente (Sperry y Morgan, 2010). En este orden de ideas, el presente artículo trata sobre la planificación y realización de un experimento para analizar los factores que influyen en la disposición a pagar (DAP) por un nuevo servicio de tren rápido de pasajeros de alta velocidad entre Barranquilla y Cartagena. Como se trata de un servicio que todavía no está disponible para los usuarios potenciales, se utilizará una técnica de valoración contingente como herramienta para el experimento, que por definición pretende simular un mercado a través de encuestas a usuarios potenciales (Riera, 1994), con el fin de saber cuál sería la DAP de los usuarios de este nuevo servicio.

Después de presentar el planteamiento, la estructura de este artículo es la siguiente. En primer lugar, se muestra la caracterización de la investigación que identifique los factores que tienen un efecto significativo sobre la DAP. En segundo lugar, se presenta el experimento factorial final y el ajuste del modelo de regresión. En los tres casos se verifica la adecuación de los modelos propuestos. Posteriormente, se realiza el análisis de los resultados y esto conduce a la optimización del diseño del sistema de transporte. Por último, se presentan las principales conclusiones del documento.

\section{CARACTERIZACIÓN DEL EXPERI-}

\section{MENTO $2^{4}$}

En general, el diseño de $2^{k}$ se utiliza en las etapas iniciales del trabajo experimental ya que proporciona el menor número de recorridos de los factores $k$ que se pueden estudiar en un diseño factorial completo (Montgomery, 2013). En este caso, el trabajo presentado se encuentra en el contexto del análisis de sistemas de transporte, en particular en el diseño de sistemas basados en el transporte público de pasajeros. Geográficamente, el estudio es parte de la prestación de servicios de transporte entre Barranquilla - Cartagena, teniendo en cuenta la existencia (hipotéticamente hablando) de un servicio ferroviario de alta velocidad entre las dos ciudades.

\subsection{Diseño}

Como se trata de un nuevo servicio de transporte, se considera que se puede hacer el análisis a través de la experimentación. Inicialmente, los objetivos que se quieren alcanzar están relacionados con la posibilidad de identificar los factores más importantes que influyen en la DAP de los usuarios potenciales del servicio de tren de alta velocidad. Generalmente, la DAP por los servicios de transporte se asocia con factores tales como el tiempo de viaje, intervalo del servicio y el nivel del servicio (Jaimes y Márquez, 2009), pero recientemente también se ha estudiado el efecto de fiabilidad (Zheng et al., 2010; Xie et al., 2012) al igual que el efecto de riesgo de accidente (Rheinberger y Bründl, 2008), entre otros factores.

Recientemente, algunos estudios han abordado la influencia de los servicios de Wi-Fi y conexiones eléctricas en los usuarios para decidir viajar en tren, encontrando que existe una influencia positiva que puede llegar incluso a un aumento del $36 \%$ de la demanda declarada de servicios actuales (Mokhtarian et al., 2013, Ettema et al., 2012) y se genera una mayor disposición a pagar por los servicios nuevos (Maccagni, 2012). 
Se consideraron cuatro factores en el experimento: tres cuantitativos y uno cualitativo, como se muestra en la Tabla 1. En comparación con la modalidad de bus, en el cual el tiempo de viaje es de 200 minutos, el tren de alta velocidad ahorra tiempo considerablemente, como se ve en el rango propuesto que varía entre 60 y $90 \mathrm{~min}$. En este experimento en particular, el efecto del riesgo de accidente no se tuvo en cuenta debido a que es difícil considerar la naturaleza cualitativa y actitudinal de seguridad (Daziano, 2012).

De acuerdo con estudios previos sobre vías, se espera que el intervalo de servicio promedio del tren sea de una hora (SITR Mobility Consortium, 2014) y por esta razón se decidió experimentar con niveles de 30 y $90 \mathrm{~min}$. Dado que la longitud de trayectoria estimada es de $135 \mathrm{~km}$, suponiendo una velocidad promedio de $110 \mathrm{~km} / \mathrm{h}$, se esperaría tener un tiempo de viaje de $75 \mathrm{~min}$, así que se considera razonable utilizar valores de 60 y $90 \mathrm{~min}$ en el caso de este factor.

La fiabilidad del tiempo de viaje para los servicios ferroviarios suele ser alta, por lo que no se espera una variación grande. Es razonable pensar en una variación de $15 \%$ sobre el tiempo de viaje promedio (Li et al., 2010) y por esa razón se proponen valores de 5 y 20 minutos para fines experimentales.

\begin{tabular}{|c|c|c|c|}
\hline \multicolumn{4}{|c|}{$\begin{array}{l}\text { TABLA 1. FAC } \\
\text { EXPERIMENTAL }\end{array}$} \\
\hline Factor & Descripción & Código & Nivel \\
\hline \multirow{2}{*}{ A } & \multirow{2}{*}{$\begin{array}{l}\text { Intervalo del servicio } \\
\text { (min) }\end{array}$} & - & 30 \\
\hline & & + & 90 \\
\hline \multirow{2}{*}{ B } & \multirow{2}{*}{ Tiempo de viaje (min) } & - & 60 \\
\hline & & + & 90 \\
\hline \multirow{2}{*}{ C } & \multirow{2}{*}{$\begin{array}{l}\text { Variación en el tiempo } \\
\text { de viaje (min) }\end{array}$} & - & 5 \\
\hline & & + & 20 \\
\hline \multirow{2}{*}{ D } & \multirow{2}{*}{$\begin{array}{l}\text { Wi-Fi y Tomas eléc- } \\
\text { tricas }\end{array}$} & - & No \\
\hline & & + & Yes \\
\hline
\end{tabular}

La variable de respuesta del experimento es la DAP por el nuevo servicio de transporte, en miles de pesos colombianos. En teoría, la relación entre la DAP y los factores A, B y C sugiere un efecto negativo, es decir, entre más largo el intervalo del servicio del tren o la trayectoria, menor DAP del usuario. El mismo comportamiento debe exhibir el tiempo de viaje de fluctuación (recíproco de la fiabilidad) y, como aumenta la fluctuación, se espera que la DAP disminuya, dada la pérdida de fiabilidad. Por el contrario, en el caso del Wi-Fi y los tomas eléctricas se espera un efecto positivo, ya que al tener el servicio (factor de alto nivel) implicaría un aumento en la DAP del usuario.

Es importante destacar que todos los factores que se eligieron son parte del diseño del sistema de transporte que se está estudiando, por lo que los resultados del experimento serían muy útiles en la fase de diseño. Dependiendo de las condiciones tecnológicas y de infraestructura que se elijan, se podrá lograr un tiempo de viaje más bajo o más alto. Dado el nivel fijo de demanda, el intervalo deseado entre las salidas podría determinar la capacidad de los vagones. Dependiendo de los sistemas de control que se lleven a cabo, será posible mantener la fluctuación del tiempo de viaje en determinados niveles. Del mismo modo, si la disponibilidad de Wi-Fi y tomas eléctricas tiene un efecto significativo sobre la DAP, se podrá evaluar si es posible o no ofrecer este servicio.

Dado que este es un experimento de factores de selección con 3 réplicas, se propone un total de 4 observaciones por condición experimental, es decir, un total de 64 datos, los cuales se consideran una muestra adecuada para esta primera parte del análisis. El experimento fue al azar. Se generaron números de forma aleatoria $\mathrm{y}$, antes de realizar el experimento, se construyó el sondeo sobre la base de cómo hacer las observaciones. Básicamente, el experimento consistió en presentar a cada encuestado una ficha informativa (Figura 1) de una condición experimental asociada a las características de un servicio ferroviario de alta velocidad nuevo e hipotético entre Barranquilla y Cartagena. La variable de salida corresponde a la DAP que declaró el usuario sobre la condición experimental que se presentó. 


\section{Figura 1. Listado descriptivo de la condición experi- mental en la caracterización del experimento}

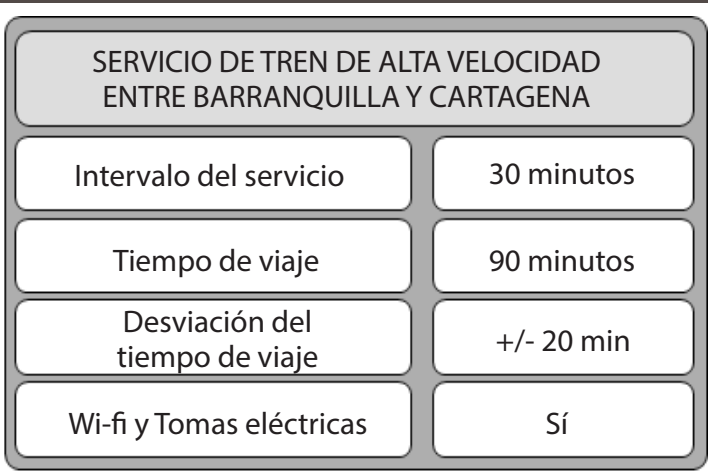

Si esta nueva alternativa de transporte estuviera en uso, por favor responda cuánto estaría dispuesto a pagar.

Puesto que Barranquilla es la ciudad principal de la ruta propuesta, el experimento sólo estudió individuos ubicados en esta ciudad principal ya que el servicio ferroviario de alta velocidad tiende a operar como un servicio express, enlazando únicamente las estaciones principales de las ciudades, sin detenerse en ninguna otra estación. Por lo tanto, como se espera que los recorridos sean de ida y vuelta, se consideró suficiente estudiar una sola ciudad.

\subsection{Aplicación y análisis del experimento}

La caracterización del experimento se llevó a cabo el 9 de mayo de 2014 en la terminal de buses en Barranquilla a través de un sistema de encuesta. Algunos usuarios del servicio de transporte público (pasajeros por carretera) entre Barranquilla - Cartagena fueron seleccionados de forma sistemática. Antes de proceder con el análisis, es necesario afirmar que la técnica utilizada para el experimento puede resultar sesgada, generalmente conocida como estratégica, lo cual se relaciona con la dificultad que los usuarios puedan encontrar revelando la verdadera DAP (Riera, 1994), lo que ha generado a algunas críticas sobre el método, especialmente cuando se trata de bienes no comercializables en el mercado. No obstante según lo anterior, al igual que en el caso estudiado, los usuarios están realmente pagando una tarifa para acceder al servicio actual y el registro que se presenta no contiene precios sugeridos, por lo tanto, se espera que les sesgo sea controlado de manera aceptable.

\begin{tabular}{|c|c|c|c|c|c|}
\hline $\begin{array}{c}\text { Fuente } \\
\text { R: Intervalo del servicio }\end{array}$ & $\begin{array}{c}\text { Suma de cuadrados } \\
534.766\end{array}$ & $\begin{array}{c}\text { Df } \\
1\end{array}$ & $\begin{array}{c}\text { Media Cuadrática } \\
534.766 \\
\end{array}$ & $\begin{array}{c}\text { F-Ratio } \\
31,20\end{array}$ & $\begin{array}{l}\text { Valor P } \\
0,0000 \\
\end{array}$ \\
\hline B: tiempo de viaje & 301.891 & 1 & 301.891 & 17,61 & 0,0001 \\
\hline C: Variación en el tiempo de viaje & 58.141 & 1 & 58.141 & 3,39 & 0,0717 \\
\hline D:Wi-Fi y Tomas eléctricas & 74.391 & 1 & 74.391 & 4,34 & 0,0426 \\
\hline$A B$ & 17.016 & 1 & 17.016 & 0,99 & 0,3241 \\
\hline $\mathrm{AC}$ & 17.016 & 1 & 17.016 & 0,99 & 0,3241 \\
\hline$A D$ & 26.266 & 1 & 26.266 & 1,53 & 0,2218 \\
\hline $\mathrm{BC}$ & 1.891 & 1 & 1.891 & 0,11 & 0,7412 \\
\hline $\mathrm{BD}$ & 1.891 & 1 & 1.891 & 0,11 & 0,7412 \\
\hline$C D$ & 0.391 & 1 & 0.391 & 0,02 & 0,8806 \\
\hline$A B C$ & 5.641 & 1 & 5.641 & 0,33 & 0,5689 \\
\hline $\mathrm{ABD}$ & 0.141 & 1 & 0.141 & 0,01 & 0,9282 \\
\hline $\mathrm{ACD}$ & 13.141 & 1 & 13.141 & 0,77 & 0,3856 \\
\hline $\mathrm{BCD}$ & 15.016 & 1 & 15.016 & 0,88 & 0,3540 \\
\hline $\mathrm{ABCD}$ & 19.141 & 1 & 19.141 & 1,12 & 0,2959 \\
\hline Error & 822.750 & 48 & 17.141 & & \\
\hline Total & 1909.480 & 63 & & & \\
\hline
\end{tabular}


El rango de la variable de respuesta estuvo entre $\$ 15.000$ y $\$ 42.000$ pesos, con un promedio de $\$ 27,23$ y la desviación estándar igual a $\$ 5,5$. Este rango de valores se considera razonable porque el pasaje de bus por la misma ruta es de $\$ 17.500$ pesos. El análisis de la varianza de la DAP (Tabla 2) indica que la fluctuación del tiempo de viaje (relacionado con el concepto de fiabilidad) no tuvo efectos significativos, a diferencia de otros factores (rango, tiempo de viaje y fluctuación) que eran verdaderamente relevantes. Los resultados del análisis de la varianza permiten suponer razonablemente que las interacciones del segundo, tercero y cuarto orden son insignificantes. Esta evidencia abre la posibilidad de utilizar diseños factoriales fraccionados de dos niveles.

No existe una sospecha de violación de supuestos. El gráfico de probabilidad normal de los residuales y las estadísticas básicas de la distribución de errores (asimetría y curtosis) indica que los residuales vienen de una distribución normal.

El análisis de autocorrelación de los residuales indica que el supuesto de independencia se cumple puesto que los errores se producen al azar en el momento. De hecho, cuando se calculó el primer intervalo se encontró que este es igual a 0,0429, lo que indica que los residuales son independientes. No hay ninguna sospecha de violación del supuesto de homocedasticidad ya que los gráficos de los residuales por factor no presentan una estructura obvia. A partir de la caracterización del experimento se llegó a la conclusión que para los usuarios potenciales del servicio, son importantes los siguientes factores: rango, tiempo de viaje y la disponibilidad de Wi-Fi y tomas eléctricas durante el viaje.

\section{EXPERIMENTO FACTORIAL FINAL}

Con base en los resultados de la sección anterior, el rendimiento del experimento factorial final fue en función del diseño de $2 \cdot 2^{3}$ que implica una variable cualitativa con 2 niveles y 2 variables cuantitativas con 3 niveles, para un total de 18 condiciones experimentales. Los niveles de cada una de las variables que representa el experimento factorial final se encuentran en la Tabla 3.

TABLA 3. FACTORES Y NIVELES DEL EXPERIMENTO FACTORIAL FINAL

\begin{tabular}{c|c|c} 
Factor & Descripción & Nivel \\
\hline$H$ & Intervalo del servicio (min) & $30,60,90$ \\
\hline$T$ & Tiempo de viaje (min) & $60,75,90$ \\
\hline W & Wi-Fi y Tomas eléctricas & No, Yes \\
\hline
\end{tabular}

\subsection{Determinación del tamaño de la} muestra

La determinación del tamaño de la muestra no parece ser un problema crítico debido a la naturaleza del experimento, cuyas observaciones se hacen a través de la aplicación de una encuesta de valoración contingente que permite recopilar información por un costo muy bajo. De hecho, como se puede ver en la Tabla 4, las principales empresas de transporte terrestre de pasajeros entre Barranquilla y Cartagena movilizan más de 1.500 pasajeros diarios. Por lo tanto, es posible tomar una muestra relativamente grande sin costos adicionales. Por factores de facilidad y costos, se decidió replicar el experimento 10 veces, de modo que fuera posible tener un total de 180 observaciones.

TABLA 4. PROMEDIO DE VIAJES Y PASAJEROS DIARIOS DE LAS PRINCIPALES COMPAÑÍAS EN LA RUTA ENTRE EN BARRANQUILLA - CARTAGENA

\begin{tabular}{|c|c|c|}
\hline Compañía de buses & Viajes & Pasajeros \\
\hline Expreso Brasilia & 28,8 & 375 \\
\hline Copetrán & 25,2 & 333 \\
\hline Colibertador & 24,7 & 315 \\
\hline La Costeña & 19,9 & 270 \\
\hline Cooperativa Simón Bolívar & 5,9 & 86 \\
\hline Berlinas del Fonce & 5,1 & 80 \\
\hline Almirante Padilla & 4,4 & 57 \\
\hline Total & 114 & 1516 \\
\hline
\end{tabular}




\section{final}

3.2 Datos y análisis del experimento

La última campaña de recolección de datos se llevó a cabo durante el 20 y 21 de mayo de 2014 en la Terminal Metropolitana de Transporte de Barranquilla con la ayuda de una empresa de sondeo, quienes entregaron a los pasajeros hojas con las condiciones experimentales para que manifestaran su disposición a pagar por el uso del nuevo servicio de transporte.

La variable de respuesta del experimento final se encuentra en el rango entre $\$ 15.000$ y $\$ 45.000$ pesos colombianos, con un promedio de $\$ 29.02 \mathrm{mil}$ pesos y una desviación estándar de 6,64. En comparación con los datos que se obtuvieron en la caracterización del experimento, los resultados encontrados aquí parecen coherentes. En este caso, tanto el valor estándar de asimetría y la curtosis estandarizada están dentro del rango esperado para los datos de una distribución normal. La distribución de la variable de respuesta se presenta en la Figura 2. Debido a la forma en que los individuos expresan su DAP, se encontraron unos valores de frecuencia más altos en múltiplos de 5 , lo cual es habitual en este tipo de clasificación.

Figura 2. Distribución de las frecuencias de la DAP en el experimento factorial final

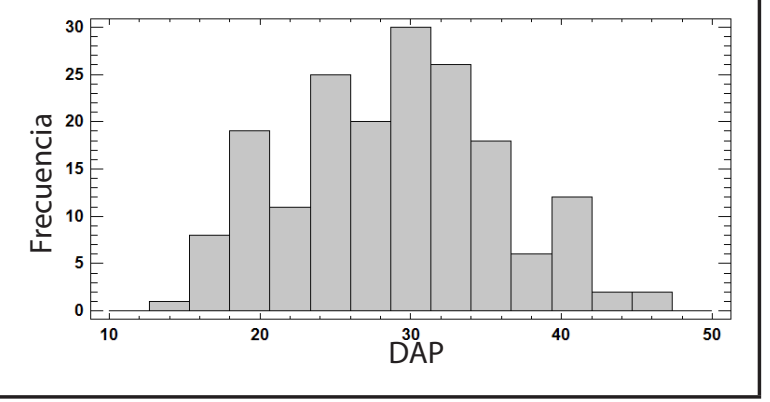

Se realizó el análisis de varianza del experimento donde se encontró que los efectos de los factores estudiados fueron significativos. Esto quiere decir que algunos niveles de los factores analizados ocasionan diferentes valores de la variable de respuesta de la DAP. Se hicieron algunos análisis de contraste utilizando la prueba de rango múltiple de la DAP para cada uno de los factores considerados con el fin de saber qué niveles de cada uno de los factores están en realidad causando esta diferencia. Las pruebas realizadas en cada par de cada variable de diseño indicaron que en todos los casos hay diferencias significativas.

La Tabla 5 muestra la media de la DAP para cada nivel de los factores. También muestra el error estándar de cada media, lo cual es una medida de la variabilidad del muestreo. Las dos columnas del extremo derecho muestran los intervalos de confianza con un $95 \%$ para cada una de las medias. Como se indicó anteriormente, los valores de la media de los niveles de cada factor son estadísticamente diferentes entre sí.

\begin{tabular}{|c|c|c|c|c|c|}
\hline \multicolumn{3}{|c|}{ TABLA 5. LA DAP PARA CADA NIVEL DE LOS FACTORES } \\
Nivel & Conteo & Media & $\begin{array}{c}\text { Error } \\
\text { Estándar }\end{array}$ & $\begin{array}{c}\text { Límite } \\
\text { Inferior }\end{array}$ & $\begin{array}{c}\text { Límite } \\
\text { Superior }\end{array}$ \\
\hline $\begin{array}{c}\text { GRAN } \\
\text { MEDIA }\end{array}$ & 180 & 29.022 & & & \\
\hline $\begin{array}{c}\text { Intervalo } \\
\text { del } \\
\text { servicio }\end{array}$ & & & & & \\
\hline 30 & 60 & 32.283 & 0.504 & 31.289 & 33.278 \\
\hline $\begin{array}{c}60 \\
90\end{array}$ & 60 & 28.850 & 0.504 & 27.855 & 29.845 \\
\hline $\begin{array}{c}\text { Tiempo } \\
\text { de viaje }\end{array}$ & 60 & 25.933 & 0.504 & 24.939 & 26.928 \\
\hline 60 & 60 & 34.567 & 0.504 & 33.572 & 35.561 \\
\hline 75 & 60 & 29.067 & 0.504 & 28.072 & 30.061 \\
\hline $\begin{array}{c}\text { Tomas } \\
\text { eléctricas }\end{array}$ & 60 & 23.433 & 0.504 & 22.439 & 24.428 \\
\hline $\begin{array}{c}\text { No } \\
\text { Wí }\end{array}$ & 90 & 27.656 & 0.411 & 26.843 & 28.468 \\
\hline & 30.389 & 0.411 & 29.577 & 31.201 \\
\hline
\end{tabular}

La verificación del supuesto de normalidad se explicó gráficamente como se muestra en la Figura 3, la cual tiende a doblar ligeramente hacia abajo desde el lado izquierdo, también implica que los residuales negativos no son tan grandes (en valor absoluto) como se esperaba. Sin embargo, este gráfico 
no muestra una desviación marcada de la distribución normal. Cuando se estaba verificando el control de la distribución de los residuales con la prueba de Kolmogórov-Smirnov se encontró que encajan de manera efectiva en una distribución normal.

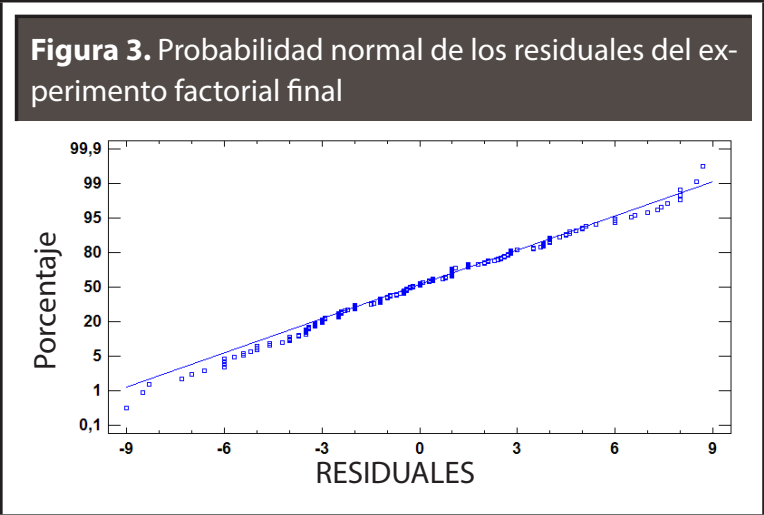

Con el objetivo de detectar las correlaciones entre los residuales, se diseñó una gráfica de los residuales en orden cronológico respecto a la toma de datos, como se muestra en la Figura 4, lo cual se muestra también en el gráfico de autocorrelación. Se puede observar que la dispersión residual no varía con el tiempo a medida que avanza el experimento, lo que permite que se cumpla el supuesto de independencia. Además, la autocorrelación del primer intervalo es igual a 0,0287 , lo cual garantiza la independencia de los residuales.

Si el modelo es correcto y los supuestos se cumplen, los residuales no deben tener una estructura; en particular, no deben estar relacionados con otra variable incluyendo la variable de respuesta (Montgomery, 2013). Al aplicar la prueba de Bartlett se descubrió que el supuesto de homocedasticidad no se violó. Debe aclararse que cuando el experimento corresponde a un modelo equilibrado y el supuesto de normalidad está verificado, la violación del supuesto de homocedasticidad no afecta significativamente la prueba $\mathrm{F}$.

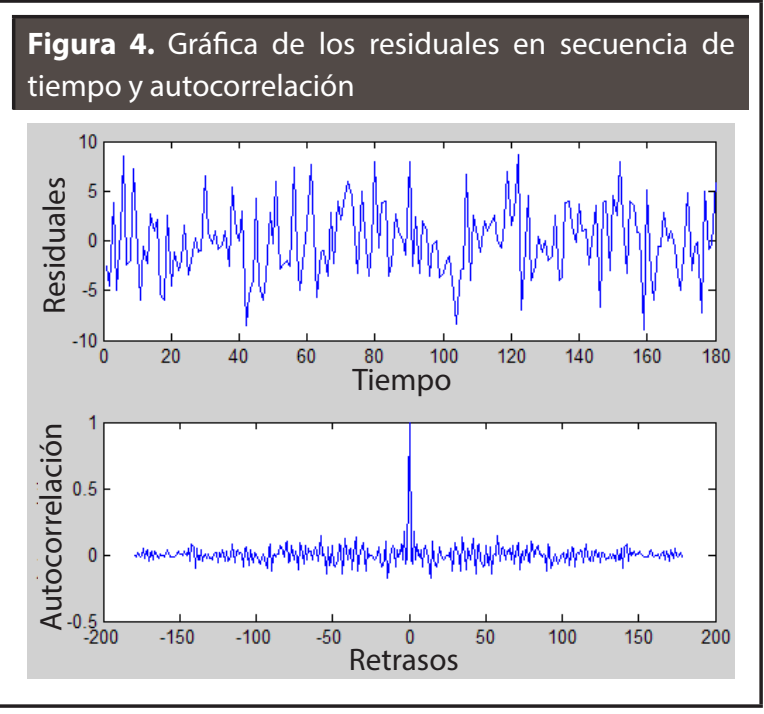

\section{RESULTADOS}

Se probaron varias especificaciones del modelo de regresión, y se encontró que el mejor modelo lineal es el que incluye la constante y los tres factores principales. La Tabla 6 muestra los resultados de ajuste de un modelo de regresión múltiple lineal para describir la relación entre la DAP y las tres variables independientes. La ecuación del modelo ajustado es

$W T P=61,839-0,106 H-0,371 T+2,733 W$

La Tabla 6 indica que el modelo presentado es significativo. Adicionalmente, de acuerdo con la estadística- $t$ se puede decir que todos los factores incluidos en el modelo son estadísticamente significativos. Además, los coeficientes estimados tienen signos correctos ya que, como se había anticipado al elegir las variables experimentales, entre mayor sea el intervalo del servicio de tren y mayor tiempo de viaje, disminuye la DAP de los usuarios. Del mismo modo, el acceso a Wi-Fi y tomas eléctricas aumenta la DAP. 


\begin{tabular}{|c|c|c|c|c|}
\hline \multicolumn{6}{|c|}{ TABLA 6. REGRESIÓN MÚLTIPLE - DAP } \\
\hline Parámetros & Estimación & $\begin{array}{c}\text { Error } \\
\text { Estándar }\end{array}$ & Estadística t & Valor-p \\
\hline CONSTANTE & 61.839 & 1.943 & 31,82 & 0,0000 \\
\hline $\begin{array}{c}\text { Intervalo del } \\
\text { servicio (H) }\end{array}$ & $-0,106$ & 0,012 & $-8,99$ & 0,0000 \\
\hline $\begin{array}{c}\text { Tiempo de } \\
\text { viaje (T) }\end{array}$ & $-0,371$ & 0,024 & $-15,78$ & 0,0000 \\
\hline $\begin{array}{c}\text { Wi-Fi y To- } \\
\text { mas eléctri- } \\
\text { cas (W) }\end{array}$ & 2.733 & 0.576 & 4,74 & 0,0000 \\
\hline
\end{tabular}

La estadística R-Cuadrado indica que el modelo como ajuste explica $66,69 \%$ de la variabilidad en la DAP. La estadística R-Cuadrado ajustada, la cual es más adecuada para la comparación de modelos con diferentes números de variables independientes es de $66,12 \%$. El error estándar de la estimación muestra la desviación estándar de los residuales siendo de 3,87. Puesto que el valor P en la Tabla 7 es menor que 0,05 , existe una relación estadísticamente significativa entre las variables en el nivel de confianza del 95,0\%. El error absoluto medio $\left(\mathrm{MAE}^{1}\right.$ ) de 3,06123 es el valor promedio de los residuales. El estadístico de Durbin-Watson (DW) ( $p=0,6971$ ) prueba los residuales para determinar si existe alguna correlación significativa en función del orden que se presenta en el archivo de datos. Como el valor P es mayor que 0,05 , no hay ningún indicador de autocorrelación serial en los residuales en el nivel de confianza de $95,0 \%$.

\begin{tabular}{|c|c|c|c|c|c|}
\hline \multicolumn{6}{|c|}{ TABLA 7. ANÁLISIS DE LA VARIANZA } \\
Fuente & $\begin{array}{c}\text { Suma de } \\
\text { cuadrados }\end{array}$ & Df & $\begin{array}{c}\text { Media } \\
\text { cuadrática }\end{array}$ & F-Ratio & Valor p \\
\hline Model & $5.264,41$ & 3 & 1754,8 & 117,45 & 0,0000 \\
\hline Residual & $2.629,50$ & 176 & 14,9404 & & \\
\hline $\begin{array}{c}\text { Total } \\
\text { (Corr.) }\end{array}$ & $7.893,91$ & 179 & & & \\
\hline
\end{tabular}

También se puede notar que los residuales no tienen una estructura respecto a ninguna de las va-

$\overline{1}$ Por sus siglas en inglés Mean Absolute Error (MAE) riables explicativas. Además, una estructura obvia de los residuales no está presente con respecto a la variable predictiva. Esta situación permite afirmar que el modelo está bien especificado y explica satisfactoriamente la DAP de los usuarios potenciales del servicio.

La Tabla 8 muestra las correlaciones estimadas entre los coeficientes en el modelo ajustado. Por lo general, estas correlaciones se pueden utilizar para detectar la presencia de multicolinealidad seria, es decir, la correlación entre las variables predictivas. En nuestro caso, no hay correlaciones con valores absolutos mayores de 0,5 (sin incluir el término constante).

\begin{tabular}{|c|c|c|c|c|}
\hline \multicolumn{4}{|c|}{ TABLA 8. MATRIZ DE CORRELACIÓN PARA LOS } \\
COEFICIENTES ESTIMADOS \\
\hline Constante & $\begin{array}{c}\text { Intervalo } \\
\text { del servicio }\end{array}$ & $\begin{array}{c}\text { Duración } \\
\text { de reco- } \\
\text { rrido }\end{array}$ & $\begin{array}{c}\text { Wi-Fi y } \\
\text { Tomas } \\
\text { eléctricas }\end{array}$ \\
\hline Constante & 1,0000 & $-0,3631$ & $-0,9078$ & $-0,1482$ \\
\hline $\begin{array}{c}\text { Intervalo } \\
\text { del servicio }\end{array}$ & $-0,3631$ & 1,0000 & 0,0000 & 0,0000 \\
\hline $\begin{array}{c}\text { Tiempo de } \\
\text { viaje }\end{array}$ & $-0,9078$ & 0,0000 & 1,0000 & 0,0000 \\
\hline $\begin{array}{c}\text { Wi-Fi y } \\
\text { Tomas } \\
\text { eléctricas }\end{array}$ & $-0,1482$ & 0,0000 & 0,0000 & 1,0000 \\
\hline
\end{tabular}

Según las pruebas estadísticas realizadas, se concluye que el modelo de regresión propuesto no debería simplificarse. Por otra parte, ya que no hay otras especificaciones probadas que hayan dado buenos resultados, se puede decir que difícilmente se podría desarrollar un mejor modelo con los datos disponibles. En la Tabla 9 se presentan los intervalos de confianza para los coeficientes del modelo de regresión. Los intervalos de confianza muestran cómo se pueden estimar de forma precisa los coeficientes, según la cantidad de datos disponibles y la interferencia que también se presenta. 


\begin{tabular}{|c|c|c|c|c|}
\hline Parámetros & Estimación & $\begin{array}{l}\text { Error } \\
\text { estándar }\end{array}$ & $\begin{array}{l}\text { Límite } \\
\text { inferior }\end{array}$ & $\begin{array}{l}\text { Límite } \\
\text { superior }\end{array}$ \\
\hline Constante & 61.839 & 1.943 & 58.003 & 65.674 \\
\hline $\begin{array}{l}\text { Intervalo } \\
\text { del } \\
\text { servicio }\end{array}$ & $-0,106$ & 0,012 & $-0,129$ & $-0,083$ \\
\hline $\begin{array}{l}\text { Tiempo de } \\
\text { viaje }\end{array}$ & $-0,371$ & 0,024 & $-0,418$ & $-0,325$ \\
\hline $\begin{array}{l}\text { Wi-Fi y } \\
\text { tomas } \\
\text { eléctricas }\end{array}$ & 2.733 & 0.576 & 1.596 & 3.870 \\
\hline
\end{tabular}

El experimento explora la DAP de los usuarios potenciales de un nuevo servicio de tren de alta velocidad entre Barranquilla y Cartagena. Está claro que, como el sistema de transporte que se está estudiando no está en funcionamiento, pensar en su optimización sería poco razonable. En cambio, los datos recogidos y el modelo calibrado pueden ser útiles para determinar la viabilidad del sistema de transporte propuesto y definir sus parámetros operacionales.

Para determinar la viabilidad del sistema de transporte propuesto, se consultó la tarifa actual de algunos trenes de alta velocidad en el mundo y asimismo se calculó la tarifa para el servicio hipotético que se podría proveer entre Barranquilla y Cartagena (Tabla 10). Se descubrió que la tarifa promedio de servicio debe ser de unos $\$ 50.000$ pesos colombianos.

Este valor, en comparación con la DAP promedio de los usuarios potenciales, que de acuerdo con el experimento es igual a $\$ 29.000$ pesos, indica que desde esta perspectiva el nuevo servicio de transporte que se está analizando sería difícilmente factible. Sin embargo, es habitual subsidiar dichos servicios (Goeverden et al., 2006); por lo que los resultados de esta investigación podrían ser utilizados para evaluar una política de subsidio que permita que este servicio sea viable. De acuerdo con estudios anteriores, parece factible justificar la existencia de un subsidio sobre la base de los beneficios de ahorro en los costos de operación del vehículo, en tiempo de viaje y la reducción de los costos externos (SITR Mobility Consortium, 2014).
TABLA 10. TARIFA EQUIVALENTE (MILES DE PESOS COLOMBIANOS) PARA EL SERVICIO DE TRANSPORTE DE ALTA VELOCIDAD ENTRE BARRANQUILLA Y CARTAGENA

\begin{tabular}{l|c|c} 
Ruta & $\begin{array}{c}\text { Distancia } \\
(\mathbf{k m})\end{array}$ & $\begin{array}{c}\text { Tarifa } \\
\text { Equivalente }\end{array}$ \\
\hline Sevilla-Mérida & 190 & 38 \\
\hline Murcia-Valencia & 229 & 45 \\
\hline Berlin-Dresde & 193 & 47 \\
\hline Madrid-Valladolid & 212 & 52 \\
\hline Milán-Venecia & 245 & 60 \\
\hline Venecia-Florencia & 258 & 64 \\
\hline
\end{tabular}

Aunque se recomienda no extrapolar más allá de la región que contiene las observaciones originales (Montgomery, 2013), el modelo de regresión ajustado indica que la DAP podría llegar a $\$ 50.000$ pesos colombianos en condiciones de funcionamiento, incluidas las conexiones Wi-Fi y tomas eléctricas, con un intervalo de 15 minutos cada uno y un tiempo de viaje de 30 minutos, lo que implica una velocidad de $250 \mathrm{~km} / \mathrm{h}$, lo que es muy difícil de lograr con la tecnología que se ha considerado hasta ahora y con las restricciones de velocidad en lugares urbanos.

Los métodos de superficie de respuesta se utilizan comúnmente para optimizar los procesos (Montgomery, 2013). En este caso, la DAP se ha modelado con éxito a través de una función lineal de las variables independientes, y se encontró un modelo de primer orden, cuyo gráfico de contorno de la superficie de respuesta se muestra en la Figura 5, suponiendo que tiene acceso a Wi-Fi y tomas eléctricas.

El modelo de regresión escrito que depende de las variables codificadas es

$$
W T P=27,656-3,175 H-5,733 T+2,733 W
$$

Teniendo en cuenta que el servicio de transporte tiene acceso a Wi-Fi y a conexiones eléctricas, el modelo se puede reescribir como:

$$
W T P=30,389-3,175 H-5,733 T
$$


En este caso, la pendiente máxima de ascenso es igual a 0,5538 y el vector de gradiente corresponde a [-3.175, -5.733]. Es evidente que, dentro del rango de operación que se consideró, el valor máximo de la DAP (\$39.132 = pesos colombianos) se alcanzaría por un recorrido de 60 minutos y un intervalo entre viajes de 30 minutos, siempre y cuando los usuarios tengan acceso a Wi-Fi y a tomas eléctricas.

La implicación de disminuir el tiempo de viaje estimado de $75 \mathrm{~min}$ a $60 \mathrm{~min}$ es simplemente para aumentar la velocidad del diseño operativo de 110 $\mathrm{km} / \mathrm{h}$ a $135 \mathrm{~km} / \mathrm{h}$, es decir, un aumento del $23 \%$ de la velocidad para disminuir el tiempo de viaje al 20\%. La necesidad de reducir el intervalo entre viajes de $60 \mathrm{~min}$ a $30 \mathrm{~min}$ es un poco más compleja, ya que al proporcionar un servicio más frecuente, implica usar equipos de menor capacidad o proporcionar el servicio con menor índice de ocupación de los vehículos. Alguna de estas condiciones operativas ocasionó un aumento de los costos de prestación del servicio, lo que afectaría negativamente las expectativas de los usuarios al tener una tarifa más baja. A pesar de lo anterior, en caso de que se establezcan las condiciones de funcionamiento del nuevo servicio de transporte con Wi-Fi y conexiones eléctricas, con un intervalo de 30 minutos y tiempo de viaje de 60 minutos, se estima que la DAP solo llega a $\$ 39.000$ pesos colombianos, aun lejos de los $\$ 50.000$ pesos colombianos que se calcularon en función de los referentes internacionales.

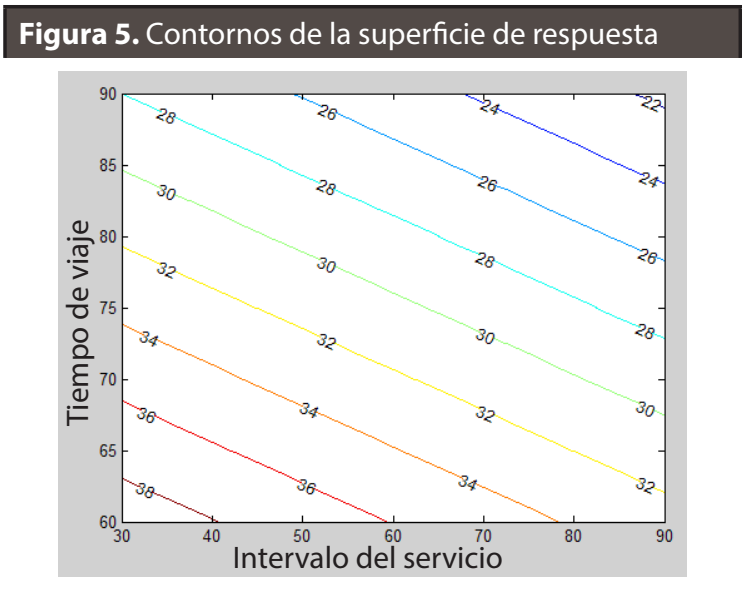

\section{CONCLUSIONES}

Los legisladores en todo el mundo han considerado el desarrollo de infraestructuras ferroviarias de alta velocidad como una posible estrategia para resolver el problema del transporte de pasajeros entre ciudades. Como se describe en este artículo, a pesar de la importante inversión, los ferrocarriles de alta velocidad podrían reducir las externalidades ambientales. En Colombia, los servicios de trenes de pasajeros de alta velocidad no se están ofreciendo, pero esta idea ha sido considerada en algunos itinerarios tales como la ruta entre Barranquilla y Cartagena.

Se ha estudiado experimentalmente la disposición a pagar (DAP) de los usuarios potenciales de un servicio hipotético de trenes de pasajeros de alta velocidad entre Barranquilla y Cartagena. A partir de la caracterización del experimento se encontró que la variación en el tiempo de viaje asociada con la fiabilidad del servicio de transporte no produce un efecto significativo sobre la DAP de los usuarios potenciales, es decir, aquellos que actualmente utilizan el servicio de transporte público entre las dos ciudades.

A través del uso de un experimento factorial basado en 180 observaciones, se encontró que el intervalo entre oficinas, el tiempo de viaje y el acceso a Wi-Fi y tomas eléctricas tienen un efecto significativo sobre la DAP de los usuarios. Además, mediante la realización de contrastes entre pares de cada una de las variables de diseño se encontró que en todos los casos hay diferencias significativas entre los niveles considerados para cada factor.

Se calibró un modelo de regresión de primer orden, el cual explica satisfactoriamente la DAP en términos de variables de intervalo, tiempo de viaje y Wi-Fi y las conexiones eléctricas. El modelo de regresión se utilizó para optimizar el diseño del sistema, concluyendo que dentro del rango de operación considerado, el valor máximo de la DAP se alcanzaría con un recorrido de 60 minutos y un intervalo de servicio del tren de 30 minutos, siempre y cuando los usuarios tengan acceso a Wi-Fi y a conexiones de eléctricas. Bajo estas condiciones de funcionamiento, la DAP 
sería $\$ 39.000$ pesos colombianos, en comparación con una tarifa de $\$ 50.000$ pesos estimados a partir de los referentes internacionales, lo cual pone en duda la viabilidad del sistema propuesto.

\section{RECONOCIMIENTO}

Agradecemos al Gobierno del Departamento del Atlántico y al Sistema General de Regalías por haber financiado parcialmente este trabajo a través de Proyectos LOGPORT, BPIN 20120001001911050672.

\section{REFERENCIAS}

Albalate, D.; Bel, G.; Fageda, X. (2015). Competition and cooperation between high-speed rail and air transportation services in Europe. Journal of Transport Geography 42, pp. 166-174.

Chen, Z.; Xue, J.; Rose, A.Z.; Haynes, K.E. (2016). The impact of high-speed rail investment on economic and environmental change in China: A dynamic CGE analysis. Transportation Research Part A: Policy and Practice 92, pp. 232-245.

Clewlow, R.R., Sussman, J. M.; Balakrishnan, H. (2014). The impact of high-speed rail and low-cost carriers on European air passenger traffic, Transport Policy 33, pp. 136-143.

Consorcio Movilidad SITR (2014). Sistema integrado de transporte región Caribe - SITR. TPD-IN-SITR699-01: Pasajeros. Departamento Nacional de Planeación, Bogotá, 97 p.

Daziano, R. (2012). Taking account of the role of safety on vehicle choice using a new generation of discrete choice models. Safety Science 50, pp. 103-112.

De Rus, G.; Kågeson, P. (2012). Efficient solutions for passenger transport: Investing public money in High-Speed Rail. $19^{\text {th }}$ ACEA, $17 \mathrm{p}$.

Eriksson, L.; Johansson, E.; Kettaneh-Wold, N.; Wikström, C.; Wold. S. (2008). Design of experiments: Principles and applications Third revised and enlarged edition. Umetrics Academy, Sweden, $459 \mathrm{p}$.

Ettema, D.; Friman, M.; Garling, T.; Olson, L. E.; Fujii, S. (2012). How in-vehicle activities affect work commuters' satisfaction with public transport. Journal of Transport Geography 24, pp. 215-222.

Goeverden, C.; Rietveld, P.; Koelemeijer, J.; Peeters, P. (2006). Subsidies in public transport. European Transport No. 32, pp. 5-25.

Goupy, J.; Creighton, L. (2007). Introduction to design of experiments, with JMP examples. Third edition, SAS Institute Inc., SAS Campus Drive, Cary, North Carolina, 448 p.

Li, Z.; Hensher, D.A.; Rose, J. (2010). Willingness to pay for travel time reliability in passenger transport: A review and some new empirical evidence. Working paper ITLS-WP-10-05. Institute of Transport and Logistics Studies, The University of Sydney, 32 p.
Maccagni, A. (2012). NTV, Italo: Using Wi-Fi and other entertainment services to enhance the travel experience on board new express trains. Presentation to the BWCS Train Communication Systems Conference, Londod, UK, June, $18 \mathrm{p}$.

Márquez, L.; Jaimes, H. (2009). Modelo de demanda para un tren de pasajeros entre Tunja y Bogotá. Revista de Ingeniería Universidad de Los Andes 30, pp. 16-24.

Márquez, L.; Cantillo, V. (2013). Evaluating strategic freight transport corridors including external costs. Transportation Planning and Technology 36 (6), pp. 529-546.

Mokhtarian, P.; Neufeld, A.; Dong, Z.; Circella, G. (2013). Did free Wi-Fi make a difference to Amtrak's Capitol corridor service? An evaluation of the impact on riders and ridership. Research report UCD-ITS-RR-13-03, Institute of Transportation Studies, University of California, Davis, California, 83 p.

Montgomery, D. C. (2013). Diseño y análisis de experimentos. Limusa Wiley, segunda edición. México, 686 p.

Polo-Navarro, L.; Cipres-Bagueste, D.; Garcia-Milla, M. (2014). Impact of an intelligent transportation system on a distribution network. Through the application of discreteevent simulation and ANOVA test. DYNA, Federación asociaciones ingenieros industriales España, Alameda de Mazarredo, 89 (2), pp. 183-191.

Rheinberger, C.; Bründl, M. (2008). Paying for Safety: A Discrete Choice Experiment to Estimate the Willingness to pay for Risk Reductions on Alpine Roads, Swiss Federal Institute for Snow and Avalanche Research SLF, Risk Management Group.

Riera, P. (1994). Manual de valoración contingente. Instituto de estudios fiscales. Madrid, $188 \mathrm{p}$.

Sperry, B.R.; Morgan, C.A. (2010). Measuring the Benefits of Intercity Passenger Rail: A Study of the Heartland Flyer Corridor. Texas Transportation Institute, $180 \mathrm{p}$.

Wilson, S. R. (2011). Statistical Design and Analysis of Experiments for Next Generation Air Transportation Research. NASA Langley Research Center, NASA Statistical Engineering Symposium, May 5, $21 \mathrm{p}$.

Xie, M.; Ng, K.M.; Habibullah, M.S.; Kolowrocki, K.; Soszynska-Budny, J. (2012). A study of safety and reliability of maritime transportation systems. Life cicle reliability and safety engineering, 1(1), pp. 35-43.

Zheng, L.; Hensher, D. A.; Rose, J. (2010). Willingness to pay for travel time reliability in passenger transport: A review and some new empirical evidence. Working paper ITLSWP-10-05, Institute of Transport and Logistics Studies, University of Sydney, 32 p.

\section{PARA CITAR ESTE ARTÍCULO / TO REFERENCE THIS ARTICLE / PARA CITAR ESTE ARTIGO /}

Márquez Díaz, L.G. (2016). Disposición a pagar por el servicio de un tren rápido entre Barranquilla y Cartagena. Revista EIA, 13(26), julio-diciembre, pp. 83-94. [Online]. Disponible en: https://doi.org/10.24050/reia.v13i26.640

Revista EIA Rev.EIA.Esc.Ing.Antioq / Universidad EIA 